\title{
Network Evolution Induced by Asynchronous Stimuli through Spike-Timing-Dependent Plasticity
}

\author{
Wu-Jie Yuan ${ }^{1,2 *}$, Jian-Fang Zhou' ${ }^{1}$, Changsong Zhou ${ }^{2,3,4 *}$ \\ 1 College of Physics and Electronic Information, Huaibei Normal University, Huaibei, China, 2 Department of Physics, Centre for Nonlinear Studies and the Beijing-Hong \\ Kong-Singapore Joint Centre for Nonlinear and Complex Systems (Hong Kong), Institute of Computational and Theoretical Studies, Hong Kong Baptist University, \\ Kowloon Tong, Hong Kong, 3 Beijing Computational Science Research Center, Beijing, China, 4 Research Centre, HKBU Institute of Research and Continuing Education, \\ Virtual University Park Building, South Area Hi-tech Industrial Park, Shenzhen, China
}

\begin{abstract}
In sensory neural system, external asynchronous stimuli play an important role in perceptual learning, associative memory and map development. However, the organization of structure and dynamics of neural networks induced by external asynchronous stimuli are not well understood. Spike-timing-dependent plasticity (STDP) is a typical synaptic plasticity that has been extensively found in the sensory systems and that has received much theoretical attention. This synaptic plasticity is highly sensitive to correlations between pre- and postsynaptic firings. Thus, STDP is expected to play an important role in response to external asynchronous stimuli, which can induce segregative pre- and postsynaptic firings. In this paper, we study the impact of external asynchronous stimuli on the organization of structure and dynamics of neural networks through STDP. We construct a two-dimensional spatial neural network model with local connectivity and sparseness, and use external currents to stimulate alternately on different spatial layers. The adopted external currents imposed alternately on spatial layers can be here regarded as external asynchronous stimuli. Through extensive numerical simulations, we focus on the effects of stimulus number and inter-stimulus timing on synaptic connecting weights and the property of propagation dynamics in the resulting network structure. Interestingly, the resulting feedforward structure induced by stimulus-dependent asynchronous firings and its propagation dynamics reflect both the underlying property of STDP. The results imply a possible important role of STDP in generating feedforward structure and collective propagation activity required for experience-dependent map plasticity in developing in vivo sensory pathways and cortices. The relevance of the results to cue-triggered recall of learned temporal sequences, an important cognitive function, is briefly discussed as well. Furthermore, this finding suggests a potential application for examining STDP by measuring neural population activity in a cultured neural network.
\end{abstract}

Citation: Yuan W-J, Zhou J-F, Zhou C (2013) Network Evolution Induced by Asynchronous Stimuli through Spike-Timing-Dependent Plasticity. PLoS ONE 8(12): e84644. doi:10.1371/journal.pone.0084644

Editor: Matjaž Perc, University of Maribor, Slovenia

Received November 12, 2013; Accepted November 25, 2013; Published December 31, 2013

Copyright: (c) 2013 Yuan et al. This is an open-access article distributed under the terms of the Creative Commons Attribution License, which permits unrestricted use, distribution, and reproduction in any medium, provided the original author and source are credited.

Funding: This work is supported by the Hong Kong Research Grant Council No. HKBU202710 (CZ), the National Natural Science Foundation of China under Grant Nos. 11275027 (CZ) and 11005047 (WJY), the Young University Teacher's Fund of Anhui Provincein China under Grant No. $2008 j q 1071$ (WJY), and the Young Fund of Huaibei Normal University under Grant No. 2013xqz17 (JFZ). The funders had no role in study design, data collection and analysis, decision to publish, or preparation of the manuscript.

Competing Interests: The authors have declared that no competing interests exist.

*E-mail: yuanwj2005@163.com (WJY); cszhou@hkbu.edu.hk (CZ)

\section{Introduction}

In neural systems, an asynchronous state characterized by arbitrarily low mean spiking correlations has been observed extensively in experiments [1,2], which offers substantial advantages for information processing and coding [2]. Especially in sensory systems, the asynchronous firings or external asynchronous stimuli (which can produce the asynchronous firings) play an important role in perceptual learning, associative memory and map development $[3,4]$. It has been experimentally found that, the sensory asynchronous stimuli are used to mediate the plasticity of neural responses for learning and memory in adults and the activity-dependent development of sensory map during a critical period of early postnatal life [3-8]. For instance, late visual stimulus is able to serve instructive role for earlier arriving auditory input in the barn owl, a highly efficient predator [5]. Neural associations between stimuli and reward expectancy in primary visual cortex can form when adult rats experience an association between visual activity (conditioned stimulus) and subsequent reward (unconditioned stimulus) [6]. A recent study has reported that the asynchronous stimuli producing binocular retinal activities in mice during a critical period in development enhance eyespecific segregation and regulate retinotopy in the developing visual system [7].

Recently, an important cognitive function, cue-triggered recall of learned temporal sequences, has been experimentally studied $[9,10]$. The cue-triggered recall of a learned temporal sequential firing has been found in neuronal ensemble of hippocampus, its surrounding cortical areas [11,12] and visual cortical circuits [13]. Importantly, the learned sequential firing can be trained in experiments by the repeated asynchronous stimuli. For example, a recent study reported that [13], a moving spot that asynchronously stimulated the neurons whose receptive fields fall along the motion path, can evoke the sequential firing of neuronal ensemble in primary visual cortex. Shortly after repeated motion conditioning, a brief flash at the starting point of the motion path can also evoke the sequential firing similar to that evoked by the moving spot. In 
this experiment, the sequence learning and cue-triggered recall become more persistent with more repeats of the stimulation of the moving spot. This indicates that, asynchronous stimuli evoked by the moving spot, play an important role in the formation of the sequence learning and recall.

In general, asynchronous stimuli can be used to mediate the activity-dependent map development during a critical period of early postnatal life and the synaptic plasticity for learning and memory in adults $[3,4,6,8,14]$. Especially, asynchronous stimuli contribute to the formation of sequence learning and recall [1113]. Many neuron scientists thought that, these functional roles result from activity-dependent synaptic plasticity between neurons $[4,5,7,8,13]$. However, the organization of structure and dynamics of neural networks due to synaptic plasticity are not well understood in the presence of asynchronous stimuli. It is clear that, the asynchronous stimuli can produce asynchronous, segregative firings of pre- and postsynaptic neurons. So far, experiments have extensively found a type of synaptic modification: spike-timing-dependent plasticity (STDP), which is highly sensitive to correlations between pre- and postsynaptic firings [1517]. Particularly, this synaptic plasticity has been widely found in sensory systems [18-21], which could contribute to learning, memory and development [4,21-23]. Therefore, it is natural to expect that STDP could play an important role in response to asynchronous stimuli.

In order to explore the role of STDP in the presence of external asynchronous stimuli, we construct a network model with local connectivity and sparseness depending on the spatial distance between neurons, and use external currents to stimulate alternately on different spatial layers. We here expect that, STDP can modify some synaptic strengths to form a feedforward network due to asymmetric pre-post spike orderings resulting from asynchronous stimulus pairings. In the resulting feedforward structure, a short stimulation current can be expected to produce wave propagation of neuronal firings, which could provide an alternative explanation for the cue-triggered recall of sequence learning. In addition, the results imply an important role of STDP in generating feedforward structure and collective propagation activity required for experience-dependent map plasticity in developing in vivo sensory pathways and cortices. Since the synaptic modification due to STDP is sensitive to correlations between pre- and postsynaptic firings $[15,16]$, the resulting feedforward structure is expected to reflect the property of STDP. In the feedforward network, directed propagation dynamics of neural activity is also expected to reflect the underlying property of STDP, which suggests an application of the analysis for examining STDP by measuring neural population activity in cultured neural network.

\section{Materials and Methods}

\section{Spatial Neural Network Model}

Recently, spatial network has been widely modeled [24-27]. Considering the locally-linked structure in real neural systems, we here construct a two-dimensional neural network model with local connectivity and sparseness, depending on the spatial distance between connected neurons. As shown in Fig. 1(a), $N$ neurons are located in lattice points of the two-dimensional square with $M$ layers, where each layer has $n=N / M$ neurons. Each pair of neurons is connected locally with probability $p=e^{-k r}$ [28] by synapse with random-chosen initial synaptic strength from 0 to 1 , where $k$ denotes the locally connecting strength and $r$ is the distance between two neurons (the side length of square is regarded as 1 here). For simplicity but without loss of generality, the model is composed of Integrate-and-Fire type neurons with chemical couplings of $\delta$ function. The dynamics of the membrane potential $V_{i}$ of neuron $i$ is described by

$\tau_{m} \frac{d V_{i}}{d t}=V_{r e s t}-V_{i}+\sum_{j=1, j \neq i}^{N} g A_{i j} W_{i j}\left(V_{E}-V_{i}\right) \delta\left(t-t_{s p}^{j}\right)+I$.

Here, we adopted the parameter values as those in Refs. $[29,30]$, which model V1 cells according to empirical observations [31]. The membrane time constant $\tau_{m}$ equals $30 \mathrm{~ms}$, the resting potential $V_{\text {rest }}$ is $-60 \mathrm{mV}$, and the reversal potentials $V_{E}$ for all the excitatory synapses are $0 \mathrm{mV}$. Each neuron $i$ integrates external stimulus $I$ and inputs coming from the connecting neurons $j$ at spike time $t=t_{s p}^{j}$. When the potential $V_{i}$ reaches the threshold value $-50 \mathrm{mV}$, the neuron $i$ emits a spike, and then the membrane potential is reset to the resting potential $V_{\text {rest }}$. Here, we do not consider the refractory period of neuronal activity in order to improve the speed of modeling. However, the qualitative results are independent of the refractory period. Also, the parameter $g=0.02$ is constant synaptic conductance, $A_{i j}(i \neq j)$ is the adjacency matrix $\left(A_{i j}=0\right.$ or 1$)$ and $W_{i j}(i \neq j)$ is the weight of synaptic strength from neurons $j$ to $i$. The synaptic modifications are subject to STDP, which will be described in the following.

\section{Spike-Timing-Dependent Plasticity}

STDP has been extensively found in experiments and has received much theoretical attention in recent years [32-36]. The temporal order of presynaptic and postsynaptic spikes determines whether the synaptic change is potentiated or depressed. The synaptic change will be potentiated if presynaptic neuron spikes before postsynaptic neuron; otherwise, it will be depressed. The changes of synaptic strength are a function of the relative timing between presynaptic spike arrival and postsynaptic firing. The smaller the lag between the spikes, the larger the change. The modification $\Delta W_{i j}$ of synaptic weight $W_{i j}$ from neuron $j$ to $i$ is approximated by exponential functions of the spiking time difference $\Delta t$ between post- and presynaptic neurons [15],

$$
\left\{\begin{array}{cc}
\Delta W_{i j}=A_{+} e^{-\Delta t / \tau_{+}}, \quad(\Delta t \geq 0) \\
\Delta W_{i j}=-A_{-} e^{\Delta t / \tau_{-}}, \quad(\Delta t<0)
\end{array}\right.
$$

The parameters $A_{+}, A_{-}, \tau_{+}$and $\tau_{-}$describe the property of STDP. Generally, STDP can be divided into two forms in numerical studies. If $A_{+}=A_{-}$and $\tau_{+}=\tau_{-}$, it is temporally symmetric form; otherwise, it is temporally asymmetric form. According to Eq. (2), the synaptic dynamics in Eq. (1) is described as,

$$
\left\{\begin{array}{cl}
\Delta W_{i j}(t)=A_{+} e^{-\left(t_{s p}^{i}-t_{s p}^{j}\right) / \tau_{+}}, & \left(t_{s p}^{i} \geq t_{s p}^{j}\right) \\
\Delta W_{j i}(t)=-A_{-} e^{-\left(t_{s p}^{i}-t_{s p}^{j}\right) / \tau_{-}}, & \left(t_{s p}^{i}>t_{s p}^{j}\right)
\end{array}\left(t=t_{s p}^{i}\right),\right.
$$

when neuron $i$ fires. For simplicity of simulation and without loss of generality, we only consider the low-frequency stimuli. Thus, we here consider only the largest modification corresponding to last spike time $t_{s p}^{j}$ of neuron $j$ at spiking time $t=t_{s p}^{i}$ of neuron $i$ $\left(t_{s p}^{i} \geq t_{s p}^{j}\right)$. Particularly, at $t=t_{s p}^{i}=t_{s p}^{j}$ (i.e., synchronous spikes of 

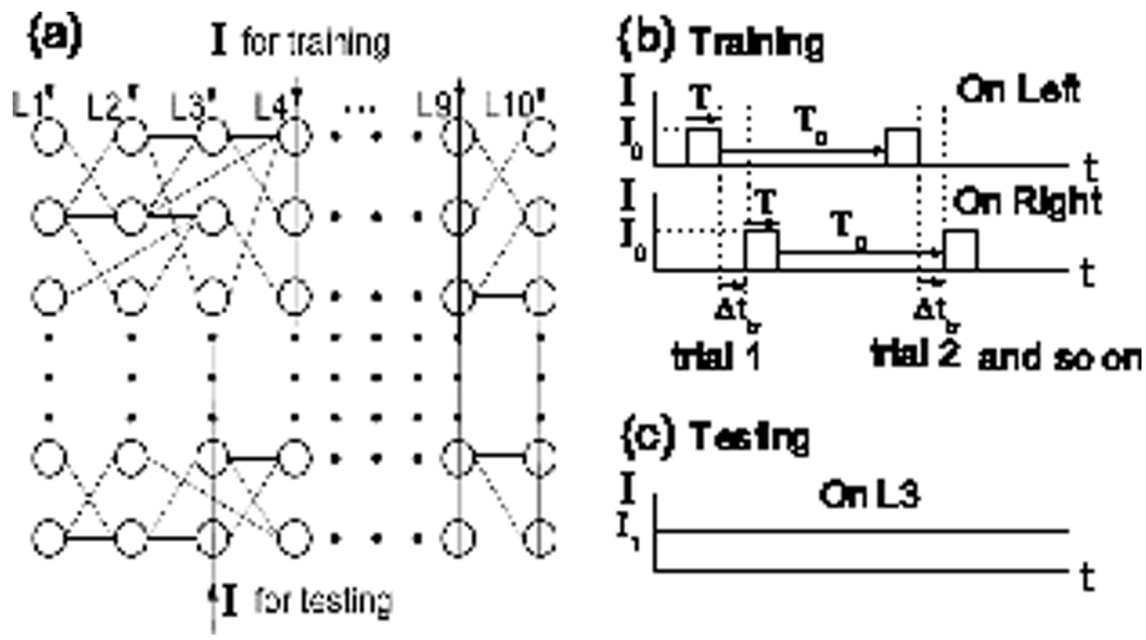

Figure 1. The locally-linked neuronal network model and external asynchronous stimulus currents. (a) The locally-linked neuronal network model on two-dimensional square having $N$ neurons and $M$ layers. Here, we take $N=1000, M=10$ and label neural number (1 - 1000) from left to right layers. (b) To train the network, the input pulse current $I_{0}$ with duration $T$ is injected alternately into each pair of layers with the left-right sequence having the same inter-stimulus interval $\Delta t_{t r}$, respectively. After each training trial, there is a long enough time $T_{0}$ to let network activity recover to the rest state for the next training. We perform training for all the pair of layers with the same number of trials. (c) To test the resulting feedforward structure and its propagative capacity, a steady current $I_{1}$ is injected into a certain layer (here, we choose the 3rd layer, i.e., L3). doi:10.1371/journal.pone.0084644.g001

neurons $i$ and $j), \Delta W_{i j}(t)$ and $\Delta W_{j i}(t)$ are both equal to $A_{+} e^{-\left(t_{s p}^{i}-t_{s p}^{j}\right) / \tau_{+}}=A_{+}$. As discussed in [15], $W_{i j}$ (or $W_{j i}$ ) is set to 0 if the change makes $W_{i j}<0$ (or $W_{j i}<0$ ) and $W_{i j}$ (or $W_{j i}$ ) is set to the maximal value 1 if the change would make $W_{i j}>1$ (or $\left.W_{j i}>1\right)$.

For STDP model, the authors only consider.

\section{External Asynchronous Stimuli}

For many years, the neural-response to external stimulus has been challenging hot topics for the study of neurology $[5,7,37,38]$. By using external asynchronous stimuli, our model produces a feedforward network due to STDP. In our model, for each pair of layers, we firstly stimulate the left layer with duration $T$ and then stimulate the corresponding right layer with the same duration $T$ after time $\Delta t_{t r}$ by inputting pulse current $I=I_{0}$ (Fig. 1(b)), where $I_{0}$ is set as a small enough value that it cannot induce spikes of neurons in other layers. Clearly, the synapses from neurons of the left layer to those of the right layer are potentiated by STDP because spikes of the left neurons precede those of the right neurons due to interval $\Delta t_{t r} \geq 0$, while the synaptic strengths of reverse connections are depressed. After each training trial for each pair of layers, there are long enough time $T_{0}$ to let network activity recover to the rest state for the next training. Here, we do the training with the same number of trials for each pair of layers. After many training trials for all the pairs of layers, all the synaptic weights from left layers to right layers will increase almost to 1 , while reverse synaptic weights will decrease approximately to 0 . Namely, the resulting network structure is expected to be a feedforward structure from left to right after many enough training trials. Then, to test the feedforward structure, we measure collective propagation property of neural activity by injecting a steady current $I_{1}$ into a certain layer in the resulting network (Fig. 1(c); here, we choose the 3rd layer).

\section{Results}

\section{Generation of Feedforward Structure}

We firstly simulate the generation of feedforward structure. As shown in Fig. 2, the initially locally-linked network (Fig. 2(a)) forms a feedforward structure (Fig. 2(b)) due to STDP by the training procedure mentioned above. Moreover, we show in Fig. 2(c) the propagation activity of neural spikes by inputting steady current. Obviously, the signals from the stimulated layer 3 (neurons $i=201$ to 300 ) spread only to the right layers (neurons $i=301$ to 1000 ), while the left layers (neurons $i=1$ to 200 ) cannot respond. This directed propagative activity indicates the formation of feedforward structure and thus reflects the existing of STDP in this neural network.

\section{Effect of Number of Training Trials}

In order to further explore the impact of the training on the resulting feedforward structure, we next investigate the dependance of synaptic weights for different connection types on the number of training trials while keeping $\Delta t_{t r}=20 \mathrm{~ms}$. Here, we divide the synaptic connections into three types: feedforward (in the training direction, from left layer to right layer in model Fig. 1(a)), feedback (the opposite training direction, from right layer to left layer) and recurrent (intra-layer) connections. Fig. 3(a) shows the situation at the beginning of our simulations, with all synaptic weights randomly chosen from 0 to 1 . After 10 trials (Fig. 3(b)), the feedforward synaptic weights are potentiated by STDP because left (presynaptic) neuron spikes before right (postsynaptic) neurons. On the contrary, the feedback synaptic weights are depressed due to reverse pre-post spike ordering. In addition, we can see the strong increase of recurrent synaptic weights. This is because the linked neurons within a layer often synchronously fire due to the same driving stimulus, causing the synaptic potentiation by STDP (see Eq. 3 for $t_{s p}^{i} \approx t_{s p}^{j}$ ). After 20 (Fig. 3(c))and 30 (Fig. 3(d)) trials, many more feedforward synapses have been strengthened (e.g. $63 \%$ being approximate to 1 in the left panel of Fig. 3(d)) and many feedback synapses have been weakened (e.g. $63 \%$ being approximate to 0 in the middle panel of 

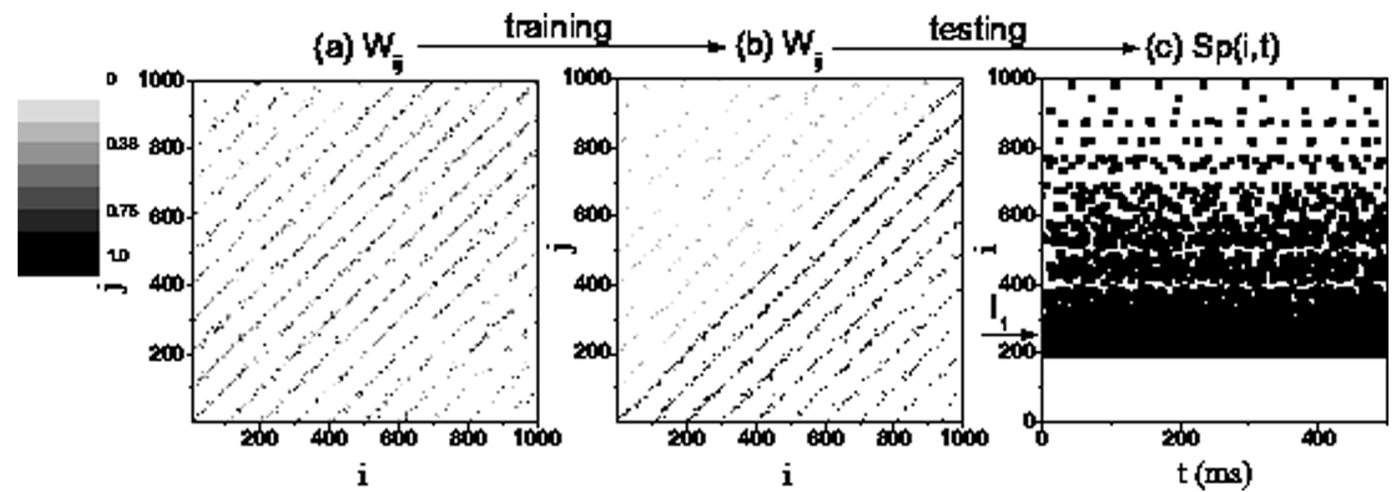

Figure 2. Evolution of the network and dynamical propagation. The weights of synaptic strengths on the model before (a) and after (b) 20 training trials. (c) The spatiotemporal pattern $S p(i, t)$ of neuron spikes by injecting steady current for testing feedforward network. The system parameters are $k=0.2, A_{+}=A_{-}=0.02$ and $\tau_{+}=\tau_{-}=50 \mathrm{~ms}$. The parameters for training (Fig. $\left.1(\mathrm{~b})\right)$ and testing (Fig. $\left.1(\mathrm{c})\right)$ are given by $I_{0}=20$, $T=100 \mathrm{~ms}, T_{0}=3 \mathrm{~s}, \Delta t_{t r}=0 \mathrm{~ms}$, and $I_{1}=400$.

doi:10.1371/journal.pone.0084644.g002

Fig. 3(d)), which makes the network form feedforward structure (Fig. 2(b)).

Fig. 4(a) shows clearly the changes of average synaptic weights with respect to the number of training trials. Obviously, the increasing amount of synaptic modification for feedforward connections is equal to the decreasing amount of synaptic modification for feedback connections (Fig. 4(a)), which results from the temporally symmetric form of STDP adopted here (i.e.,
$A_{+}=A_{-}$and $\tau_{+}=\tau_{-}$). For temporally asymmetric form of STDP, we also show the similar changes of average synaptic weights in Fig. 4(b). As in the symmetric case, the feedforward structure will emerge and become stronger with the increase of the number of training trials. Differently, the incremental amount of synaptic strengths for feedforward connections and reductive amount of synaptic strengths for feedback connections are not equal (Fig. 4(b)) owing to the asymmetric modification between
Feedforward
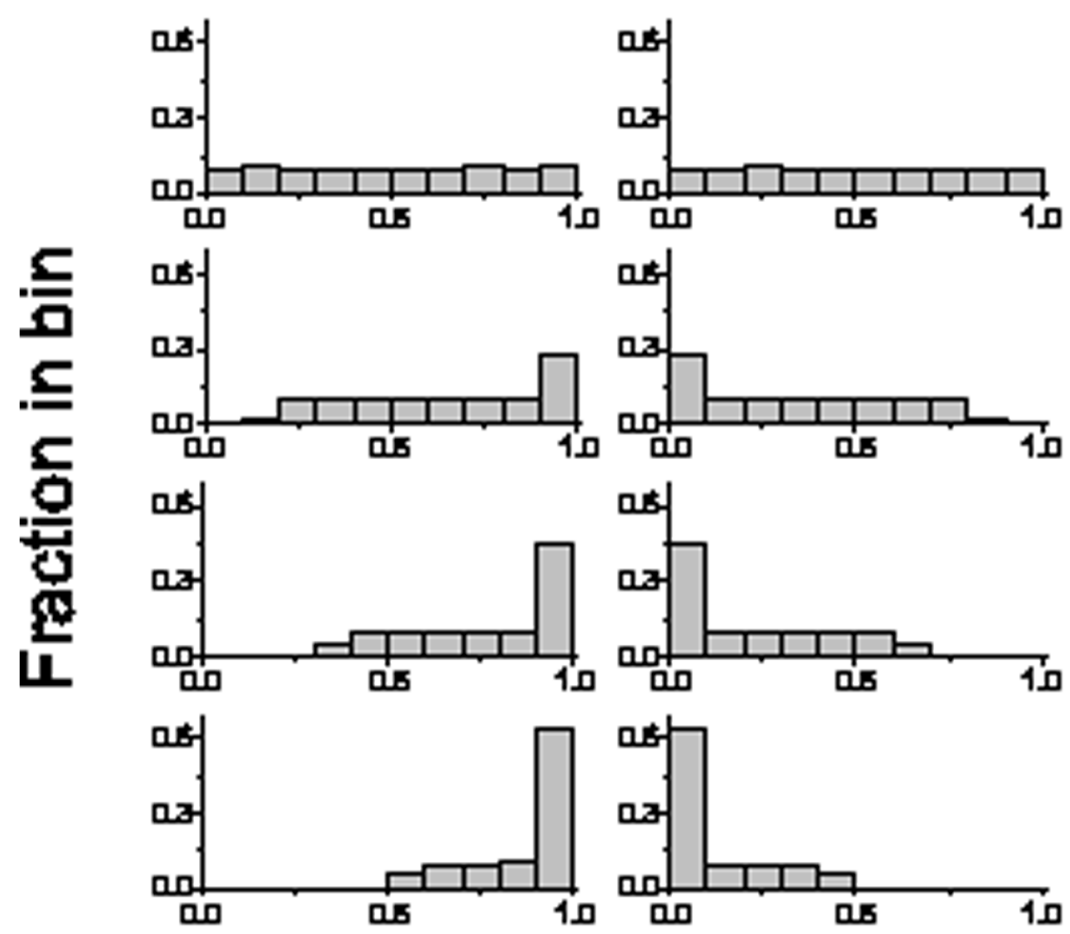

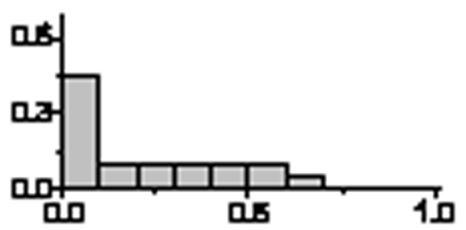

Feedback
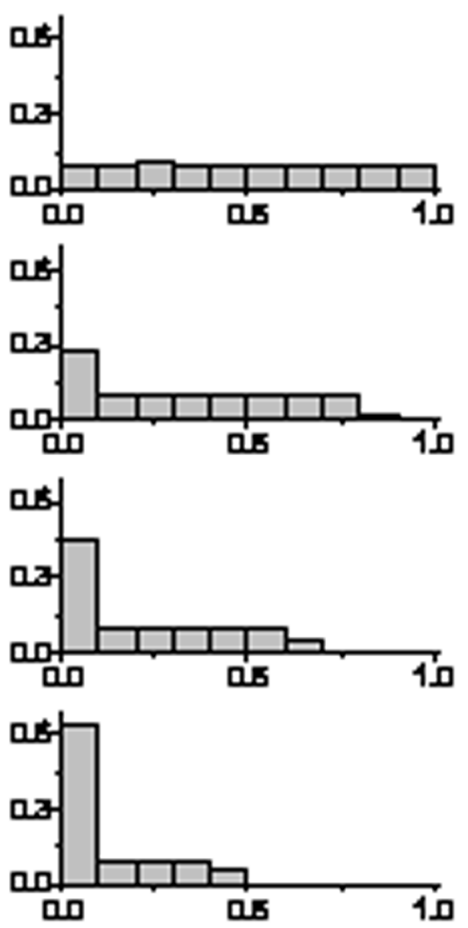

Recurrent
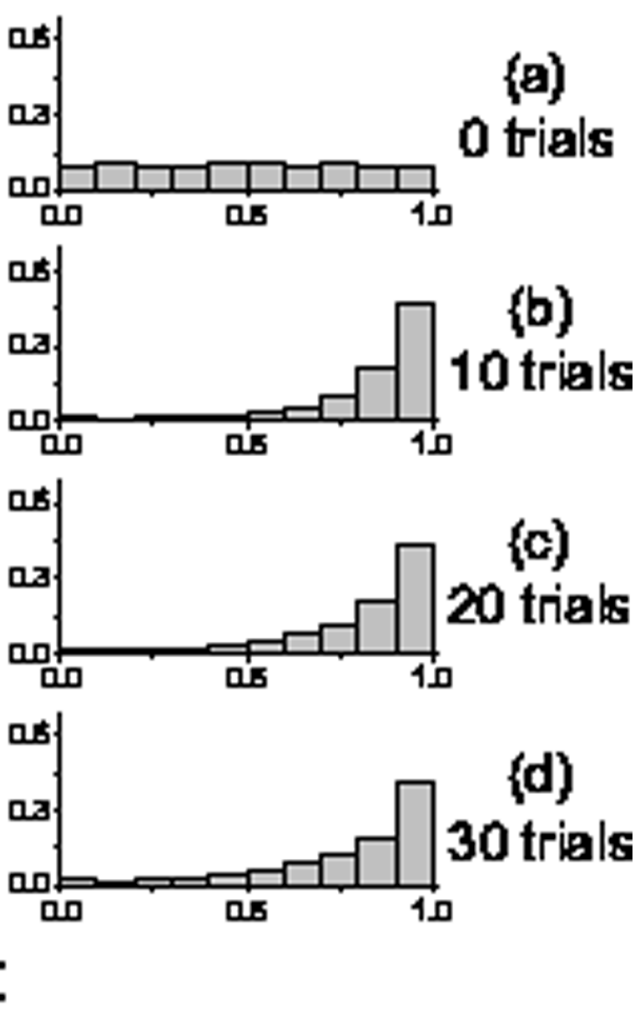

Figure 3. Distribution of synaptic weights after the different training trials. Distribution of synaptic weights for three different connection types: feedforward (left), feedback (middle) and recurrent (right) at the beginning of training (a) and after the training with different number of trials 10 (b), 20 (c), 30 (d). The same parameters are the same as in Fig. 2, except for $\Delta t_{t r}=20$ ms.

doi:10.1371/journal.pone.0084644.g003 


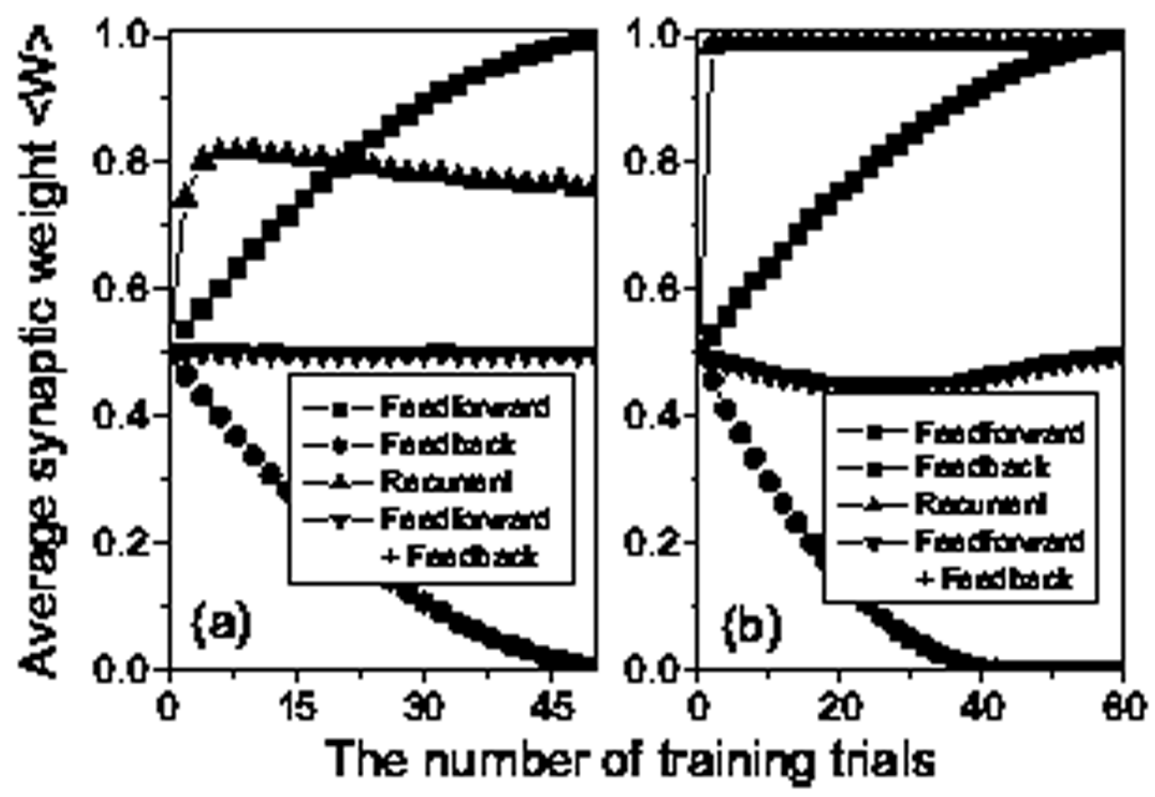

Figure 4. Average synaptic weights as a function of the number of training trials. Average synaptic weights for three different connection types: feedforward (squares), feedback (circles) and recurrent (upward triangles) as a function of the trial number for temporally symmetric form of $\operatorname{STDP}\left(A_{+}=A_{-}=0.02\right.$ and $\left.\tau_{+}=\tau_{-}=50 \mathrm{~ms}\right)$ (a) and for temporally asymmetric form of STDP $\left(A_{+}=0.04, A_{-}=0.02\right.$ and $\tau_{+}=30 \mathrm{~ms}$, $\left.\tau_{-}=60 \mathrm{~ms}\right)$ (b). The other parameters are the same as in Fig. 2 except for $\Delta t_{t r}=20 \mathrm{~ms}$. Also, we show the average synaptic weights for the connections including both feedforward and feedback types (downward triangles) in (a) and (b). doi:10.1371/journal.pone.0084644.g004

potentiated and depressed synaptic strengths with the same prepost firing timing.

\section{Effect of Inter-stimulus Interval}

We also studied the effect of inter-stimulus timing (i.e., interval $\left.\Delta t_{t r}\right)$ on synaptic weights for different connection types. When the interval is shortened to $0 \mathrm{~ms}$ (Fig. 5(a)), about $62 \%$ of feedforward synapses have been strengthened to 1 (see the left panel of Fig. 5(a)), while $62 \%$ of feedback synapses have been weakened to 0 (see the middle of Fig. 5(a)) after 20 trials, indicating a generation of strong feedforward structure (see Fig. 2(b)). With the increasing of interstimulus interval (Figs. 5(b)-5(d)), the resulting distributions of synaptic weights become broader, showing that the feedforward structure is weaker. For example, when the inter-stimulus interval is increased to $60 \mathrm{~ms}$, there are insufficient potentiation (only $23 \%$ being approximate to 1 in the left panel of Fig. 5(d)) and insufficient depression (only $23 \%$ being approximate to 0 in the middle panel of Fig. 5(d)) after 20 trials to form feedforward structure.

The right panels of Fig. 5 show that the synaptic distribution in recurrent connections appears to be independent of inter-stimulus interval. Since the training current $I_{0}$ is set as a small enough value that it cannot induce spikes of neurons in other layers without receiving training, the amount of recurrent synaptic modification within layer is completely determined by duration of external current stimuli imposed to the layer (i.e., the product of $T$ and number of training trials in Fig. 1(b)), independent of the interstimulus interval. This result implies that the synaptic modifications for the recurrent and feedforward/feedback connections are independent. So, the effect of recurrent connections on the formation of feedforward network can be neglected in our model.

Fig. 6(a) clearly shows the changes of average synaptic weights with respect to the inter-stimulus interval. Importantly, the amount of average feedforward modification relative to its initial value 0.5 (see the value at 0 trials in Fig. 4(a)) exhibits an exponential decay with $\Delta t_{t r},\langle W\rangle-0.5 \sim e^{-\Delta t_{t r} / \tau_{+}^{S}}$ with an exponent $\tau_{+}^{S}$ (Fig. 6(b)), and the amount of modification of average feedback connections relative to its initial value 0.5 (see the value at 0 trials in Fig. 4(a)) is also characterized as an exponential decay $0.5-\langle W\rangle \sim e^{-\Delta t_{t r}} / \tau_{-}^{S}$ with another exponent $\tau_{-}^{S}$ (Fig. 6(c)). For the temporally symmetric form of STDP, the two exponents $\tau_{+}^{S}$ and $\tau_{-}^{S}$ are equal (Figs. 6(b) and 6(c)) due to the equal amounts of synaptic potentiation and depression for a prepost firing timing. The two exponents $\tau_{+}^{S}$ and $\tau_{-}^{S}$ are different for the temporally asymmetric form of STDP because the symmetric modification of potentiation and depression is broken, shown in Fig. 7.

We numerically examined the relationships between the exponents $\tau_{+}^{S}$ and $\tau_{-}^{S}$ characterizing the exponential falloffs with the exponents $\tau_{+}$and $\tau_{-}$in the STDP functions (Figs. 8(a) and 8(b)). The exponents $\tau_{+}^{S}$ and $\tau_{-}^{S}$ are close to $\tau_{+}$and $\tau_{-}$, respectively. Moreover, $\tau_{+}^{S}$ and $\tau_{-}^{S}$ are independent of $\tau_{-}$and $\tau_{+}$, respectively. It's further found that, these equal relationships hold for networks with different connecting parameter $k$ (results not shown). These findings suggest that the dependence of the structure modification on inter-stimulus interval $\Delta t_{t r}$ reflects the exponents of STDP. Since network structure determines dynamics of the network, a similar dependance of population dynamics on interval $\Delta t_{t r}$ is naturally expected to reflect the underlying STDP properties in the resulting feedforward networks, which is described below.

\section{Propagation Property}

In brain, information and cognitive processing requires signal propagation through multiple regions [39]. The feedforward network plays a crucial role in signal propagation [40]. Here we study the propagation property in the above feedforward network after training. In Fig. 9(a), we show the effect of inter-stimulus 
Feedforward

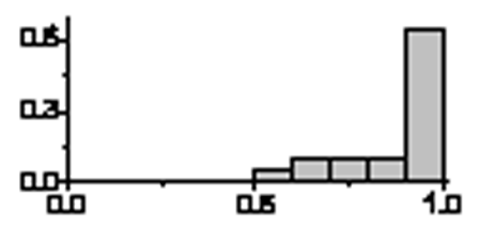

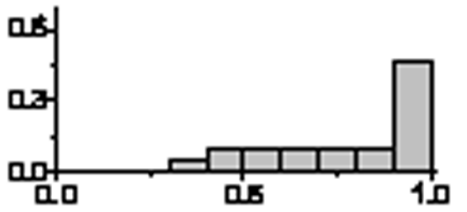

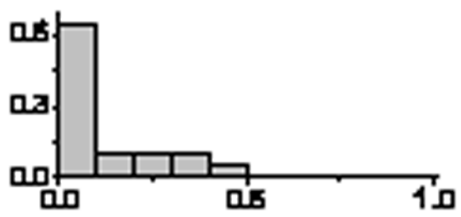

Feedback
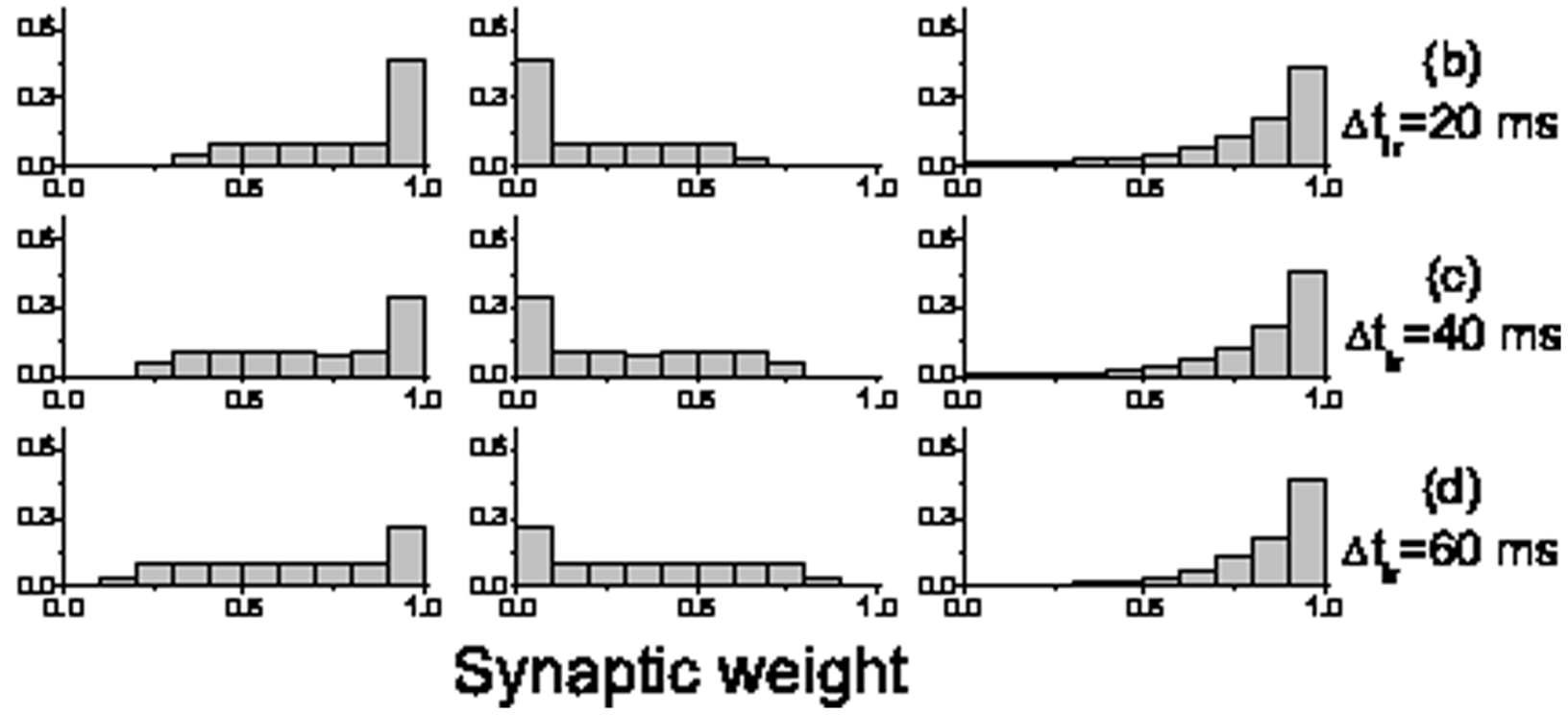

Figure 5. Distribution of synaptic weights after the different training stimulus intervals. Distribution of synaptic weights for different connection types: feedforward (left), feedback (middle) and recurrent (right) after the 20 training trials with different stimulus intervals $\Delta t_{t r}=0$ ms (a), $\Delta t_{t r}=20 \mathrm{~ms}$ (b), $\Delta t_{t r}=40 \mathrm{~ms}$ (c), $\Delta t_{t r}=60 \mathrm{~ms}$ (d). The other parameters are the same as in Fig. 2.

doi:10.1371/journal.pone.0084644.g005
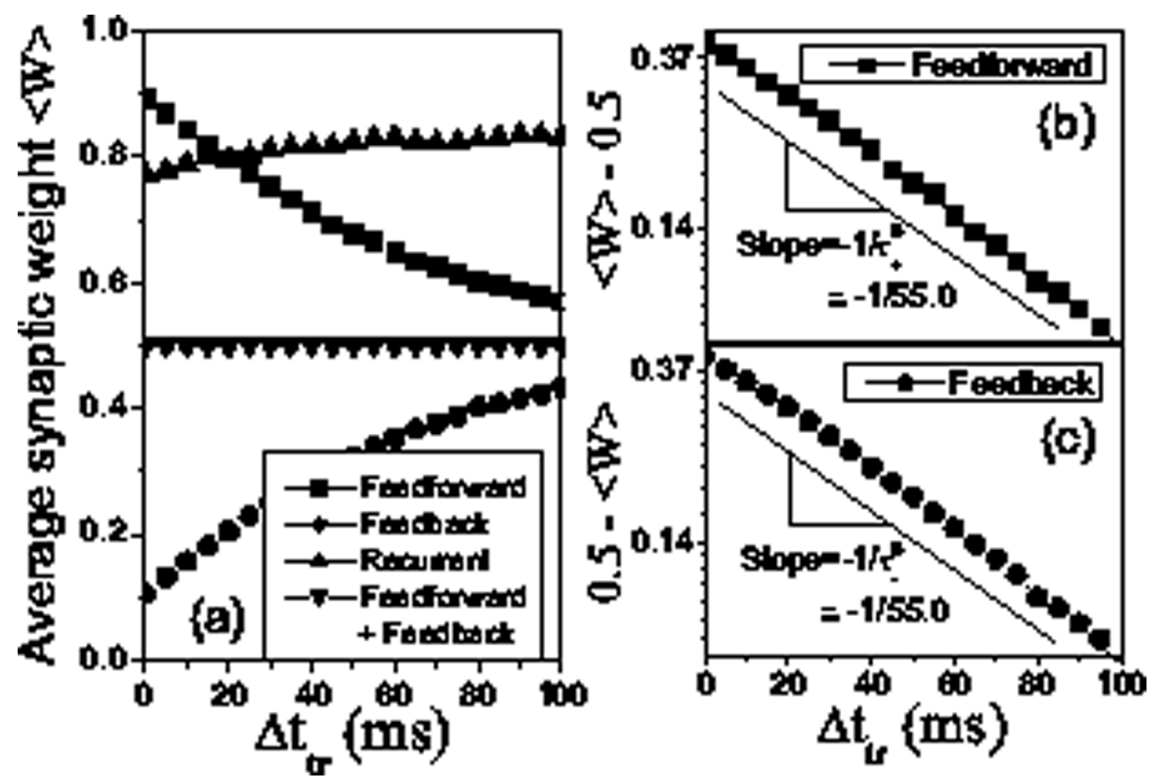

Figure 6. Average synaptic weights as a function of the stimulus interval for STDP with temporal symmetry. Average synaptic weights for three different connection types: feedforward (squares), feedback (circles) and recurrent (upward triangles) as a function of the stimulus interval after the 20 training trials (a). The amounts of average synaptic modification relative to its initial value $0.5,\langle W\rangle-0.5$ (b) and $0.5-\langle W\rangle$ (c) for feedforward (b) and feedback (c) connections exhibit exponential falloffs as the stimulus interval $\Delta t_{t r}$ increases with exponents $\tau_{+}^{S}$ (b) and $\tau_{-}^{S}$ (c), respectively in linear-log scales. we also show the average synaptic weights for the connections including both feedforward and feedback types (downward triangles) in (a). The parameters are the same as in Fig. 2.

doi:10.1371/journal.pone.0084644.g006 

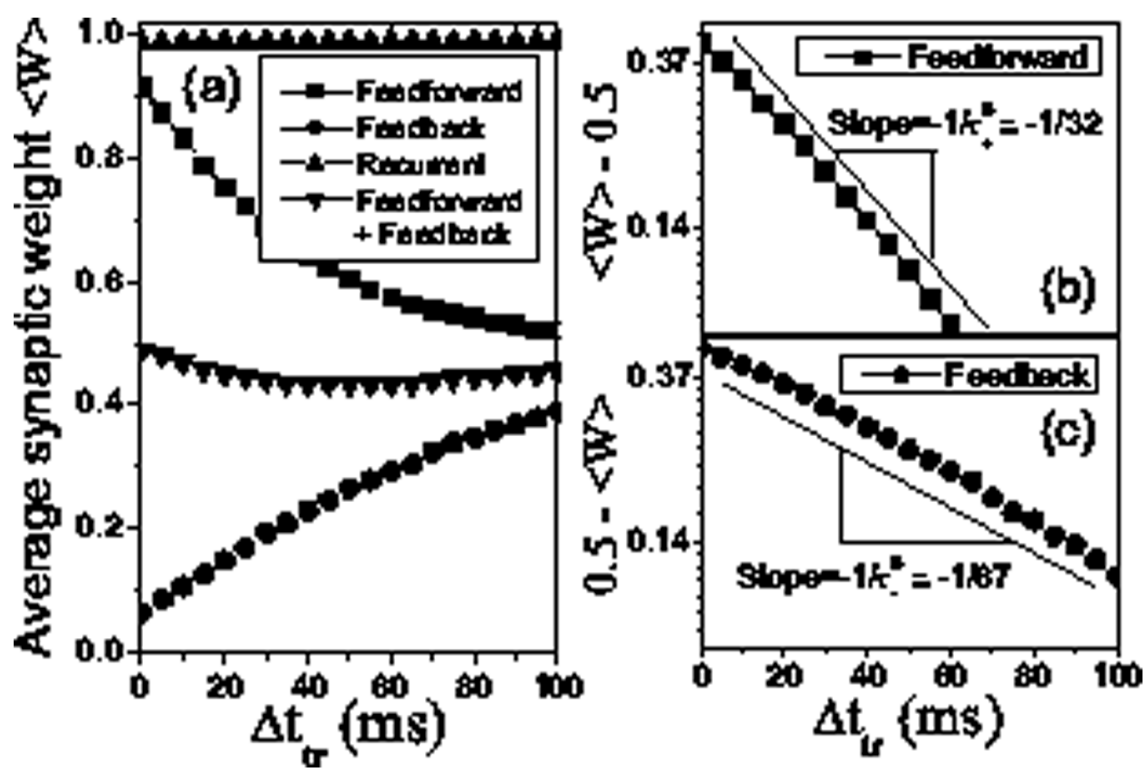

Figure 7. Average synaptic weights as a function of the stimulus interval for STDP with temporal asymmetry. The same as in Fig. 6, but for the temporally asymmetric form of STDP. Here, the parameters are the same as in Fig. 2 , except for $A_{+}=0.04, \tau_{+}=30 \mathrm{~ms}, \tau_{-}=60 \mathrm{~ms}$. doi:10.1371/journal.pone.0084644.g007

interval of training on the propagation ability. As we see in Fig. 3(c), the resulting network can support directed propagation of activity from left to right when the 3rd layer is stimulated, and the activity decays out. Here we do not consider further effect of STDP during propagation. We plot the number of spikes $N_{s p}$ of all propagated layers (i.e., layers 4 to 10 ) as a function of interstimulus interval $\Delta t_{t r}$. Clearly, it exhibits an exponential falloff $N_{s p} \sim e^{-\Delta t_{t r} / \tau_{+}^{D}}$ with exponent $\tau_{+}^{D}$, similar to the exponential falloff of STDP in Eq. (3). The exponent $\tau_{+}^{D}$ increases almost linearly with the exponent $\tau_{+}$in STDP, which is almost independent of the other exponent $\tau_{-}$(Fig. 9(b)). This is because the spike number
$N_{s p}$ of propagated layers depends mainly on the amount of feedforward synaptic modification (not on that of feedback synaptic modification), determined by the exponent $\tau_{+}$of potentiation term in Eq. (3) (Fig. 8(a)). For a comparison, we find that the scaling of the propagative activity remains the same when the feedback connections are cut (Fig. 9(a) without feedback), showing that the feedback connections are so weak that their effect on neural activity can be neglected. Additionally, the propagative activity is still almost the same without recurrent connections (Fig. 9(a)), indicating that the recurrent connections within layers also have little effect on the propagative dynamics. This is because recurrent connections are very sparse with $\mathrm{k}=0.2$ (Fig. 3(b))
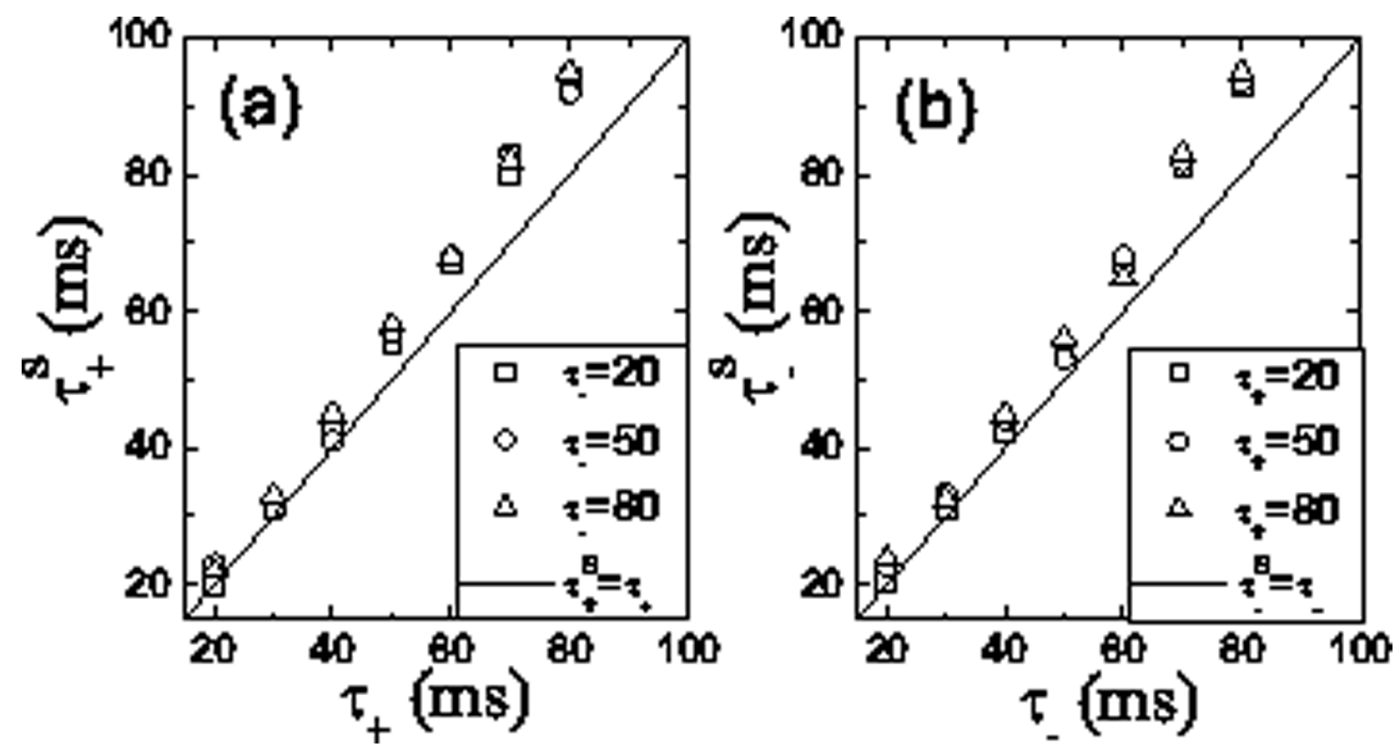

Figure 8. Dependance of exponents for the resulting structure on exponents of STDP after 20 training trials. (a) $\tau_{+}^{S}$ vs. $\tau_{+}$for different $\tau_{-}$(b) $\tau^{S}$ vs. $\tau_{-}$for different $\tau_{+}$. For comparison, $\tau_{+}^{S}=\tau_{+}$and $\tau^{S}=\tau_{-}$are shown as solid lines in (a) and (b), respectively. The other parameters are the same as in Fig. 2.

doi:10.1371/journal.pone.0084644.g008 
although recurrent connection strengths are strong. Consequently, the resulting feedforward structure governs the propagative property of the whole network in the presence of the input current. In Fig. 9(c) we show the linear relation $\tau_{+}^{D} \approx f \tau_{+}^{S}$ between the structure modification and dynamical propagation. The coefficient $f$ depends strongly on the stimulation strength $I_{1}$. As shown in Fig. 9(d), $f$ increases with $I_{1}$. From these simulation results, we can see that a STDP-structure-dynamics relation $\tau_{+} \approx \tau_{+}^{S} \propto \tau_{+}^{D}$ (Figs. 8(a), 9(b) and 9(c)) can emerge in the feedforward network induced by asynchronous stimuli on different spatial neuronal layers.

\section{Discussion}

In experiments, cultured neuronal networks and developing neural systems can both generate spontaneous activity in the form of synchronized bursting events (SBEs) [41-43], short time events during which most of the recorded neurons fire rapidly, with several subgroups. Besides, neural activity often exhibits avalanches with some dynamical clusterings described by synchronous firing of neurons in vivo and in vitro [42,44-46]. In SBEs and avalanche activity, the different dynamical clusterings behave inter-cluster asynchronous firings. So, the dynamical clusterings might strongly shape the network structure due to STDP, as the external asynchronous stimuli do in our model. This suggests an important role of STDP in generating feedforward structure and collective propagation activity on neural systems in vivo and in vitro $[23,41,44,45]$. The role of STDP in the formation of feedforward structure driven by asynchronous stimuli might provide insight into important role of propagating feedforward (from low-level to high-level brain areas) information flow for experience-dependent map plasticity in the development of in vivo sensory pathways and cortices $[4,7,21,22]$.

The sequence learning and recall may result from activitydependent synaptic plasticity. The conditioning-evoked sequential spiking of neighboring neurons is well suited for the induction of STDP [13], which selectively potentiate the directed connections between neurons and facilitate spike propagation in the same direction. Our study may provide an insight into formation mechanism of the sequence learning and recall. In our model, the resulting feedforward structure could denote sequence memory formed by the sequence learning. The spiking propagation in the direction of feedforward structure could reflect dynamical process of the cue-triggered recall. From Figs. 3 and 4, we can see the resulting feedforward structure will become clearer and so produce the more prominent propagation, as the number of our training trials increases. Indeed, with more repeats of stimulus sequence in experiment, the sequence learning and recall become more persistent [13]. Thus, our study suggests that STDP may modify neuronal structure and dynamics induced by external asynchronous stimuli to support the sequence learning and recall, which is consistent well with the experimental observation.

The amount of synaptic potentiation or depression due to STDP is typically measured by pairing a number of pre- and
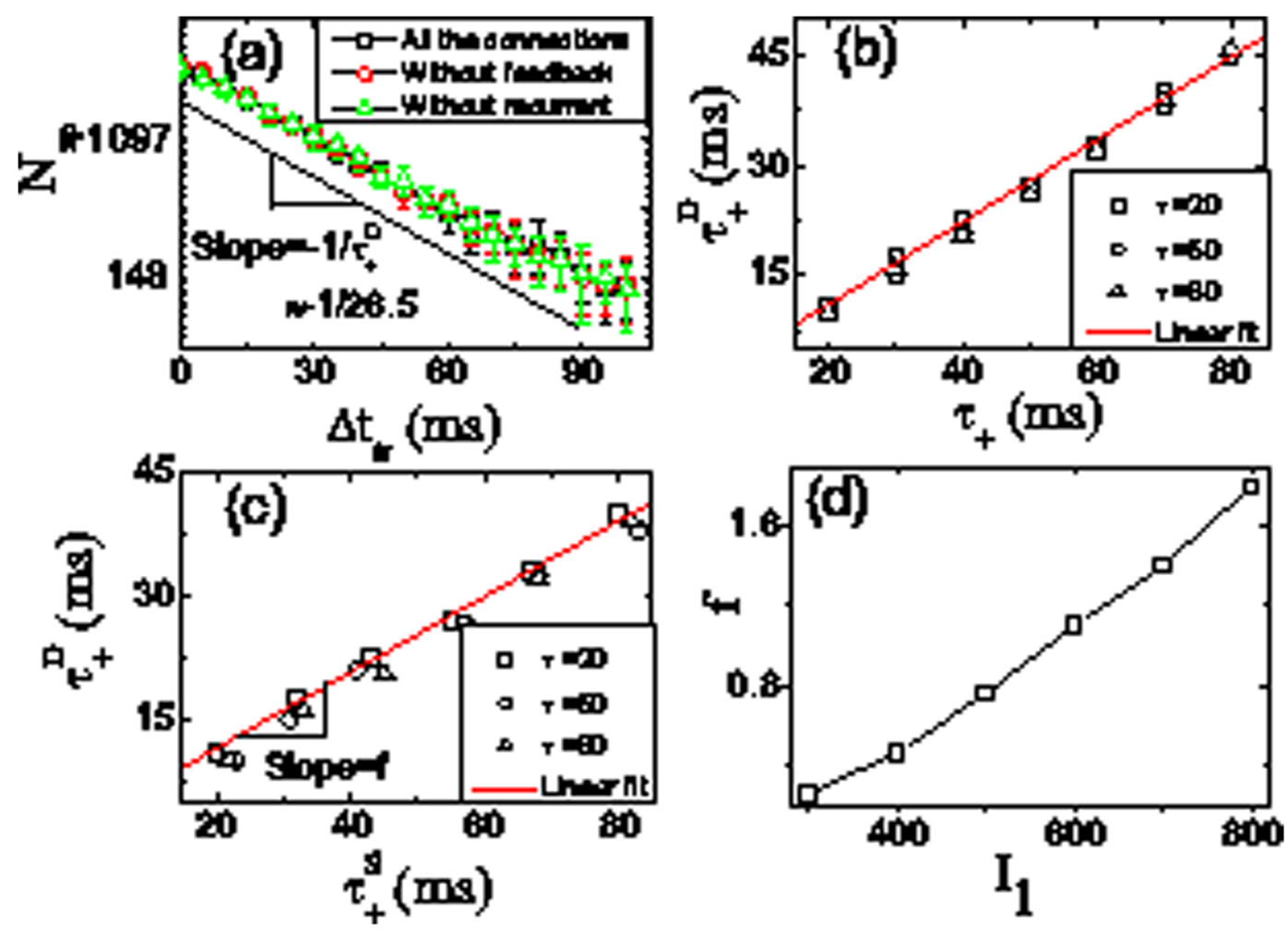

Figure 9. (Color online) The property of dynamical propagation in the resulting network. (a) The total number of spikes $N_{s p}$ of propagated layers as a function of inter-stimulus interval $\Delta t_{t r}$ in the feedforward network obtained after 20 trials of training when keeping all the connections, indicating the exponential behavior of propagation dynamics with exponent $\tau_{+}^{D}$ in the linear-log scales. For comparison, the $N_{s p}$ are also plotted in (a) when feedback and recurrent connections are cut (i.e., without feedback and without recurrent), respectively. (b) The exponent $\tau_{+}^{D}$ as a function of exponent $\tau_{+}$in STDP for different $\tau_{-}$. (c) The exponent $\tau_{+}^{D}$ as a function of the corresponding exponent $\tau_{+}^{S}$ in the feedforward network structure for different $\tau_{-}$. In (b) and (c), the linear fit lines of data are also given with slope $f$. (d) The coefficient $f$ as a function of stimulation strength $I_{1}$. The other parameters are the same as in Fig. 2. Data of $N_{s p}$ are compiled for $1000 \mathrm{~ms}$, and averaged over 10 independent runs with error bar in (a). doi:10.1371/journal.pone.0084644.g009 
postsynaptic action potentials with a specified time interval between them $[32,33,35,47]$. But, for a large-scaled neural system, it becomes very complex and difficult to directly test each synaptic modification due to spike timing and ordering. In our model, the resulting STDP-structure-dynamics relation (Figs. 8(a), 9(b) and 9(c)) suggests a potential method to examine STDP in large-scaled neural network. Our results demonstrate the propagative activity exponentially decays with exponent $\tau_{+}^{D} \propto \tau_{+}$, which provides an application for examining STDP by measuring neural population activity in a cultured neural network trained by external asynchronous stimuli. Cultured neural networks cultivated on multi-electrode arrays provide relatively simple and well-controlled model systems for investigating long-term activity of individual neurons at different locations [33,41,48-50]. By cultivating our two-dimensional network model and adopting our training/testing method, the strength of exponent $\tau_{+}$in STDP might be qualitatively examined by measuring propagation property in the resulting feedforward network. This study could shift the attention in the measurement of synaptic plasticity from the single synapse to the collective network activity. In the future, how to design other model and method for examining more parameters (e.g., $A_{+}, A_{-}$and $\tau_{-}$) of STDP is an interesting question to pursue.

To sum up, using a simple locally linked network of integrateand-fire neurons with STDP, we showed that it can naturally evolve into a feedforward structure when a pair of external asynchronous stimuli is used to train the network. Interestingly, the amount of synaptic modification due to asynchronous stimulus pairing with a time interval falls off exponentially as the interval

\section{References}

1. Ecker A, Berens P, Keliris G, Bethge M, Logothetis N, et al. (2010) Decorrelated neuronal firing in cortical microcircuits. Science 327: 584-587.

2. Renart A, De la Rocha J, Bartho P, Hollender L, Parga N, et al. (2010) The asynchronous state in cortical circuits. Science 327: 587-590.

3. Tsodyks M, Gilbert C (2004) Neural networks and perceptual learning. Nature 431: 775-781.

4. Feldman D, Brecht M (2005) Map plasticity in somatosensory cortex. Science 310: 810-815.

5. D'Souza P, Liu S, Hahnloser R (2010) Perceptron learning rule derived from spike-frequency adaptation and spike-time-dependent plasticity. Proceedings of the National Academy of Sciences of the United States of America 107: 47224727.

6. Shuler M, Bear M (2006) Reward timing in the primary visual cortex, Science 311: 1606-1609.

7. Zhang J, Ackman J, Xu H, Crair M (2012) Visual map development depends on the temporal pattern of binocular activity in mice. Nature Neuroscience 15 : 298-307.

8. Drew P, Abbott L (2006) Extending the effects of spike-timing-dependent plasticity to behavioral timescales. Proceedings of the National Academy of Sciences of the United States of America 103: 8876-8881.

9. Lisman J, Redish AD (2009) Prediction, sequences and the hippocampus. Philosophical Transactions of the Royal Society B: Biological Sciences 364: 1193-1201.

10. Fortin NJ, Agster KL, Eichenbaum HB (2002) Critical role of the hippocampus in memory for sequences of events. Nature Neuroscience 5: 458-462.

11. Gelbard-Sagiv H, Mukamel R, Harel M, Malach R, Fried I (2008) Internally generated reactivation of single neurons in human hippocampus during free recall. Science 322: 96-101.

12. Lehn H, Steffenach HA, van Strien NM, Veltman DJ, Witter MP, et al. (2009) A specific role of the human hippocampus in recall of temporal sequences. The Journal of Neuroscience 29: 3475-3484

13. Xu S, Jiang W, Poo MM, Dan Y (2012) Activity recall in a visual cortical ensemble. Nature Neuroscience 15: 449-455.

14. Yuan WJ, Zhou JF, Li Q, Chen DB, Wang Z (2013) Spontaneous scale-free structure in adaptive networks with synchronously dynamical linking. Physical Review E 88: 022818.

15. Song S, Miller K, Abbott L (2000) Competitive hebbian learning through spiketiming-dependent synaptic plasticity. Nature Neuroscience 3: 919-926.

16. Babadi B, Abbott L (2010) Intrinsic stability of temporally shifted spike-timing dependent plasticity. PLoS computational biology 6: e1000961.

17. Dan Y, Poo MM (2006) Spike timing-dependent plasticity: from synapse to perception. Physiological Reviews 86: 1033-1048. increases, similar to the falloff of STDP as the interspike interval increases. Moreover, exponents $\left(\tau_{+}^{S}\right.$ and $\left.\tau_{-}^{S}\right)$ of the exponential falloff are almost the same as the exponents $\left(\tau_{+}\right.$and $\left.\tau_{-}\right)$of STDP (i.e., $\tau_{+}^{S} \approx \tau_{+}$and $\tau_{-}^{S} \approx \tau_{-}$). Most interestingly, propagative activity also exhibits a similar exponential falloff with exponent $\tau_{+}^{D} \propto \tau_{+}$in the resulting feedforward network. Thus, by measuring the exponential falloff of the propagation activity as a function the inter-stimulus intervals in the resulting trained network, we are able to extract a direct measurement of the characteristic time scale in the network's STDP property. Generally, STDP-structuredynamics relation $\tau_{+} \approx \tau_{+}^{S} \propto \tau_{+}^{D}$ can emerge in our model. Although the formation of feedforward structure under the action of STDP has been widely studied $[51,52]$, the external asynchronous stimuli observed and used extensively in experiments, has not yet been investigated to induce the feedforward structure through STDP. The STDP-structure-dynamics relation is the first to be shown in the resulting feedforward network by imposing asynchronous stimuli. Our model may prove useful for understanding the relation between structure and dynamics induced by asynchronous stimuli through STDP and provide a starting point for studying the property of STDP by using external asynchronous stimuli.

\section{Author Contributions}

Conceived and designed the experiments: WJY CZ. Performed the experiments: WJY JFZ. Analyzed the data: WJY JFZ CZ. Contributed reagents/materials/analysis tools: WJY JFZ CZ. Wrote the paper: WJY JFZ CZ.

18. Schuett S, Bonhoeffer T, Hübener M (2001) Pairing-induced changes of orientation maps in cat visual cortex. Neuron 32: 325-337.

19. Yao H, Dan Y (2001) Stimulus timing-dependent plasticity in cortical processing of orientation. Neuron 32: 315-323.

20. Fu Y, Djupsund K, Gao H, Hayden B, Shen K, et al. (2002) Temporal specificity in the cortical plasticity of visual space representation. Science 296 : 1999-2003.

21. Mu Y, Poo M (2006) Spike timing-dependent ltp/ltd mediates visual experiencedependent plasticity in a developing retinotectal system. Neuron 50: 115-125.

22. Feldman D (2009) Synaptic mechanisms for plasticity in neocortex. Annual Review of Neuroscience 32: 33-55.

23. Yuan WJ, Zhou C (2011) Interplay between structure and dynamics in adaptive complex networks: emergence and amplification of modularity by adaptive dynamics. Physical Review E 84: 016116.

24. Perc M (2005) Spatial decoherence induced by small-world connectivity in excitable media. New Journal of Physics 7: 252.

25. Sun X, Perc M, Lu Q, Kurths J (2008) Spatial coherence resonance on diffusive and small-world networks of Hodgkin-Huxley neurons. Chaos 18: 023102.

26. Wang Z, Wang Z, Zhu XD, Arenzon JJ (2012) Cooperation and age structure in spatial games. Physical Review E 85: 011149.

27. Zhang HF, Liu RR, Wang Z, Yang HX, Wang BH (2011) Aspiration-induced reconnection in spatial public-goods game. Europhysics Letters 94: 18006.

28. Kaiser M, Hilgetag C (2004) Spatial growth of real-world networks. Physical Review E 69: 036103.

29. Abbott L, Varela J, Sen K, Nelson S (1997) Synaptic depression and cortical gain control. Science 275: 220-224.

30. Chance F, Nelson S, Abbott L (1998) Synaptic depression and the temporal response characteristics of $\mathrm{v} 1$ cells. The Journal of Neuroscience 18: 4785-4799.

31. Varela J, Sen K, Gibson J, Fost J, Abbott L, et al. (1997) A quantitative description of short-term plasticity at excitatory synapses in layer $2 / 3$ of rat primary visual cortex. The Journal of Neuroscience 17: 7926-7940.

32. Markram H, Lübke J, Frotscher M, Sakmann B (1997) Regulation of synaptic efficacy by coincidence of postsynaptic aps and epsps. Science 275: 213-215.

33. Bi G, Poo M (1998) Synaptic modifications in cultured hippocampal neurons: dependence on spike timing, synaptic strength, and postsynaptic cell type. The Journal of Neuroscience 18: 10464-10472.

34. Abbott L, Nelson S (2000) Synaptic plasticity: taming the beast. Nature Neuroscience 3: 1178-1183.

35. Froemke R, Dan Y (2002) Spike-timing-dependent synaptic modification induced by natural spike trains. Nature 416: 433-438.

36. Izhikevich E, Desai N (2003) Relating stdp to bcm, Neural Computation 15: $1511-1523$. 
37. Yuan WJ, Luo XS, Wang BH, Wang WX, Fang JQ et al. (2006) Excitation properties of the biological neurons with side-inhibition mechanism in smallworld networks. Chinese Physics Letters 23: 3115-3118.

38. Yuan WJ, Luo XS, Jiang PQ (2007) Study under AC stimulation on excitement properties of weighted small-world biological neural networks with side-restrain mechanism. Communications in Theoretical Physics 47: 369-373.

39. Vogels T, Rajan K, Abbott L (2005) Neural network dynamics. Annual Review of Neuroscience 28: 357-376.

40. Dayan P, Abbott L (2001) Theoretical neuroscience: Computational and mathematical modelling of neural systems. Cambridge, MA: MIT Press.

41. Zhan X, Lai P, Chan C (2011) Effects of glial release and somatic receptors on bursting in synchronized neuronal networks. Physical Review E 84: 011907.

42. Hulata E, Baruchi I, Segev R, Shapira Y, Ben-Jacob E (2004) Self-regulated complexity in cultured neuronal networks. Physical Review Letters 92: 198105.

43. Butts D, Kanold P, Shatz C (2007) A burst-based hebbian learning rule at retinogeniculate synapses links retinal waves to activity-dependent refinement. PLoS Biology 5: 0651-0661.

44. Shew W, Yang H, Petermann T, Roy R, Plenz D (2009) Neuronal avalanches imply maximum dynamic range in cortical networks at criticality. The Journal of Neuroscience 29: 15595-15600.

45. Hahn G, Petermann T, Havenith M, Yu S, Singer W, et al. (2010) Neuronal avalanches in spontaneous activity in vivo. Journal of Neurophysiology 104: $3312-3322$
46. Santos G, Gireesh E, Plenz D, Nakahara H (2010) Hierarchical interaction structure of neural activities in cortical slice cultures. The Journal of Neuroscience 30: 8720-8733.

47. Bi G, Rubin J (2005) Timing in synaptic plasticity: from detection to integration. Trends in Neurosciences 28: 222-228.

48. Maeda E, Robinson H, Kawana A (1995) The mechanisms of generation and propagation of synchronized bursting in developing networks of cortical neurons. The Journal of Neuroscience 15: 6834-6845.

49. Segev R, Benveniste M, Hulata E, Cohen N, Palevski A, et al. (2002) Long term behavior of lithographically prepared in vitro neuronal networks. Physical Review Letters 88: 118102.

50. Jia L, Lai P, Chan C (2007) Neuronal network growth: Model and experiment. International Journal of Modern Physics B 21: 4111-4116.

51. Gilson M, Burkitt AN, Grayden DB, Thomas DA, van Hemmen JL (2009) Emergence of network structure due to spike-timing-dependent plasticity in recurrent neuronal networks. I. Input selectivity-strengthening correlated input pathways. Biological Cybernetics 101: 81-102.

52. Takahashi YK, Kori H, Masuda N (2009) Self-organization of feed-forward structure and entrainment in excitatory neural networks with spike-timingdependent plasticity. Physical Review E 79: 051904. 